Pacific Journal of Mathematics

A CHARACTERIZATION OF LOCALLY MACAULAY 


\section{A CHARACTERIZATION OF LOCALLY MACAULAY COMPLETIONS}

\section{CRAig Huneke}

\section{rem. \\ The purpose of this note is to prove the following theo-}

Theorem 1.1. Let $(R, m)$ be a Noetherian local ring of dimension $d \geqq 1$ and depth $d-1$. By $\hat{R}$ denote the completion of $R$ in the $m$-adic topology. Then the following are equivalent: $S_{d-1}$

(1) $\hat{R}$ is equidimensional and satisfies Serre's property

(2) $H_{m}^{d-1}(R)$ has finite length

(3) There exists an $N>0$ such that if $x_{1}, \cdots, x_{d}$ is a sequence of elements $R$ with $h t\left(x_{i_{1}}, \cdots, x_{i_{j}}\right)=j$ for all $j$ elements subsets of $\{1, \cdots, n\}, 1 \leqq j \leqq n$, and if $m_{i} \geqq N$, $1 \leqq i \leqq d$, then $x_{1}^{m_{1}}, \cdots, x_{d}^{m_{d}}$ is an unconditioned $d$-sequence.

Recall the local ring $(S, N)$ is equidimensional if for every minimal prime divisor $p$ of zero, $\operatorname{dim} S / p=\operatorname{dim} S$.

Serre's property $S_{k}$ is that

$$
\operatorname{depth} R_{p} \geqq \min [\text { ht } p, k]
$$

for all primes $p$.

We will always denote the local cohomology functor by $H_{m}^{j}(\ldots)$ ([1]).

We recall the definition of a $d$-sequence due to this author [3].

Definition 0.1. A system of elements $x_{1}, \cdots, x_{d}$ in a commutative ring $R$ is said to be a $d$-sequence if

(1) $x_{i} \notin\left(x_{1}, \cdots, \hat{x}_{i}, \cdots, x_{d}\right)$

(2) $\left(\left(x_{1}, \cdots, x_{i}\right): x_{i+1} x_{k}\right)=\left(\left(x_{1}, \cdots, x_{i}\right): x_{k}\right)$ for $k \geqq i+1$ and $i \geqq 0$. A $d$-sequence is said to be unconditioned if any permutation of it remains a $d$-sequence.

These have been studied extensively by this author and have been useful to determine the "analytic" properties of ideals generated by them. In [3] the following was skown:

Proposition. Let $(R, m)$ be a local Noetherian ring. Then $R$ is Buchsbaum (see [10] for a definition and discussion) if and only if every system of parameters forms a d-sequence.

Thus Theorem 1.1 may be seen as a related result, characterizing rings in which "almost all" s.o.p.'s form a $d$-sequence. Independent 
of this characterization of rings with "lots" of $d$-sequences, Theorem 1.1 is the generalization of a result due to Steven McAdam [7] which in turn is related to a characterization of unmixed 2-dimensional local rings proved by Ratliff [8].

Let $(R, m)$ be a 2-dimensional local domain and let $b, c$ be a system of parameters. By $S(b, c, n)$ denote the least $k$ such that

$$
\left(b^{n}: c^{k}\right)=\left(b^{n}: c^{k+1}\right) \text {. }
$$

Recall a local ring $R$ is said to be unmixed if for each prime divisor $p$ of $(0)$ in $\hat{R}, \operatorname{dim} \hat{R} / p=\operatorname{dim} \hat{R}$.

Ratliff showed, [8],

Proposition. The following are equivalent for a 2-dimensional local domain

(1) $R$ is unmixed.

(2) $S(b, c, \ldots)$ is bounded.

(3) $R^{(1)}=\bigcap_{\mathrm{ht} p=1} R_{p}$ is a finite $R$-module.

McAdam discussed this and obtained the following improvement:

Proposition [5]. Let $(R, m)$ be as above. Then the following are equivalent:

(1) $R$ is unmixed, i.e., for all prime divisors $p$ of (0) in $\hat{R}$, $\operatorname{dim} \hat{R} / p=\operatorname{dim} \hat{R}=2$.

(2) $R^{(1)}$ is a finite $R$-module.

(3) There exists an $N$ such that for every s.o.p. $x, y$

$$
S\left(x, y,{ }_{-}\right) \leqq N \text {. }
$$

In particular, (3) is equivalent to saying for all $n \geqq N$ that $\left(x^{n}: y^{n}\right)=\left(x^{n}: y^{2 n}\right)$ and this is equivalent (in this case) to saying $x^{n}, y^{n}$ form a $d$-sequence.

To see our statement (1) is equivalent to (1) of the above proposition, note that if $\operatorname{dim} R=2$ and $R$ is a domain, then to say $R$ is unmixed is precisely to say $\hat{R}$ satisfies $S_{1}$ and is equidimensional.

Finally, we will show that $R^{(1)} / R$ is isomorphic to $H_{m}^{1}(R)$ in this case, and show that $R^{(1)} / R$ has finite length if and only if $R^{(1)}$ is a finitely generated $R$-module. Hence our Theorem 1.1 is the exact generalization of the above proposition of McAdam.

1. Proof of Theorem 1.1. For details on local cohomology we refer the reader to [1]. We note the following facts.

(1) Since $\operatorname{depth} R=d-1, H_{m}^{i}(R)=0$ if $i<d-1$.

(2) There is a canonical isomorphism, $H_{m}^{d-1}(R) \cong H_{\hat{m}}^{d-1}(\hat{R})$. 
(3) If $S$ is a complete regular local ring mapping onto $\hat{R}$ (see [6]) and $M$ is the maximal ideal of $S$, then $H_{m}^{d-1}(R) \cong H_{I I}^{d-1}(\hat{R})$ where $\hat{R}$ is regarded as an $S$-module.

(4) If $S$ is chosen as in (3), $e=\operatorname{dim} S$, and we let $E=H_{M}^{e}(S / M)=$ an injective hull of $S / M$, then

$$
\operatorname{Hom}_{S}\left(H_{m}^{j}(R), E\right) \cong \operatorname{Ext}_{S}^{e-j}(\hat{R}, S)
$$

and $H_{m}^{j}(R) \cong \operatorname{Hom}_{S}\left(\operatorname{Ext}_{S}^{e-j}(\hat{R}, S), E\right)$. This is local duality.

(5) We may compute $H_{m}^{d-1}(R)$ as follows: let $x_{1}, \cdots, x_{d}$ be an s.o.p., and consider the complex,

$$
\bigoplus_{i<j} R_{x_{1}, \cdots, \hat{x}_{i}, \cdots, \hat{x}_{j}, \cdots, x_{n}} \longrightarrow \bigoplus_{i} R_{x_{1}, \cdots, \hat{x}_{i}, \cdots, x_{n}} \longrightarrow R_{x_{1}, \cdots, x_{n}} \longrightarrow 0
$$

where the subscripts denote localization at the elements subscripted. Then $H_{m}^{d-1}(R)$ is isomorphic to the middle homology of this complex. If we denote by $\operatorname{syz}\left(x_{1}, \cdots, x_{d}\right)$ the module defined by $K / L$ where $K \subseteq R^{d}$ is the module of syzygies of $x_{1}, \cdots, x_{d}$ and $L$ is the submodule of syzygies which come from the trivial ones given by the Koszul relations, then

$$
H_{m}^{d-1}(R) \cong \lim _{\rightarrow} \operatorname{syz}\left(x_{1}^{n_{1}}, \cdots, x_{d}^{n^{2}} d\right)
$$

where if $m_{i} \geqq n_{i}$, the map

$$
\operatorname{syz}\left(x_{1}^{n_{1}}, \cdots, x_{d}^{n} d\right) \longrightarrow \operatorname{syz}\left(x_{1}^{m_{1}}, \cdots, x_{d}^{m_{d}} d\right)
$$

is defined by mapping a syzygy $\left(r_{1}, \cdots, r_{d}\right)$ of $\left(x_{1}^{n_{1}}, \cdots, x_{d}^{n} d\right)$ to the syzygy $\left(r_{1} x_{2}^{m_{2}-n_{2}} \cdots x_{d}^{m} d^{-n_{d}}, \cdots, r_{d} x_{1}^{m_{1}-n_{1}} \cdots x_{d-1}^{m}-_{-1}^{-n_{d-1}}\right)$ of $\left(x_{1}^{m_{1}}, \cdots, x_{d}^{m} d\right)$. We now turn to the proof of Theorem 1.1.

The fact (1) if and only if (2) holds is well-known but we give the details here for completeness.

We first observe that $H_{m}^{d-1}(R)$ has finite length if and only if $\operatorname{Hom}_{S}\left(H_{m}^{d-1}(R), E\right) \cong \operatorname{Ext}_{S}^{e-(d-1)}(\hat{R}, S)$ has finite length. (See [5].)

If $p$ is a prime in $S$ and $\hat{R} \cong S / I$, then if $p \nsupseteq I$

$$
\left(\operatorname{Ext}_{S}^{e-(d-1)}(\hat{R}, S)\right)_{p}=0 .
$$

Hence, $\operatorname{Ext}_{S}^{e-(d-1)}(\hat{R}, S)$ has finite length if and only if $\left.\operatorname{Ext}_{S}^{e-(d-1)}(\hat{R}, S)\right)_{p}=\operatorname{Ext}_{S_{p}}^{c-(d-1)}\left(\left(\hat{R}_{p}, S_{p}\right)=0\right.$ for all $p \supseteqq I, \quad p \neq M$.

If $i<d-1$, then since depth $\hat{R}=\operatorname{depth} R=d-1$, we see

$$
H_{\hat{m}}^{i}(\hat{R})=H_{i \nu}^{i}(\hat{R})=0
$$

and so

$$
\operatorname{Ext}_{S}^{p-i}(\hat{R}, S)=0
$$


or, otherwise put,

$$
\operatorname{Ext}_{S_{p}}^{k}\left(\hat{R}_{p}, S_{p}\right)=0
$$

for all $k \geqq e-(d-1)$ if and only if $H_{m}^{d-1}(R)$ has finite length. (Note for $k>e, \operatorname{Ext}_{S}^{k}(M, S)=0$ for all $M$.)

Since $S_{p}$ is regular,

$$
\operatorname{Sup}_{n}\left\{\operatorname{Ext}_{S_{p}}^{n}\left(\hat{R}_{p}, S_{p}\right) \neq 0\right\}+\operatorname{depth} \hat{R}_{p}=\operatorname{dim} S_{p} \text {. (See [9.]) }
$$

From this we may conclude that $H_{m}^{d-1}(R)$ has finite length if and only if $\operatorname{depth}(\hat{R})_{p}>\operatorname{dim} S_{p}-(e-(d-1))$ i.e., if and only if

$$
\operatorname{depth}(\hat{R})_{p} \geqq \operatorname{dim} S_{p}-\operatorname{dim} S+\operatorname{dim} \hat{R} .
$$

We claim that

$$
\operatorname{dim} S_{p}-\operatorname{dim} S+\operatorname{dim} \hat{R} \geqq \operatorname{dim}(\widehat{R})_{p}
$$

in any case. For since $S$ is regular, $\operatorname{dim} S=\operatorname{dim} S_{p}+\operatorname{dim} S / p$ and so the left side is just

$$
-\operatorname{dim} S / p+\operatorname{dim} \hat{R} .
$$

Thus it is enough to show

$$
\operatorname{dim} \hat{R} \geqq \operatorname{dim} S / p+\operatorname{dim}(\widehat{R})_{p}
$$

but this clearly always holds since $p$ contains $I$.

Thus we have shown $H_{m}^{d-1}(R)$ has finite length if and only if

(*) $\quad \operatorname{depth}(\hat{R})_{p} \geqq \operatorname{dim} S_{p}-\operatorname{dim} S+\operatorname{dim} \hat{R} \geqq \operatorname{dim}(\hat{R})_{p}$.

We claim these last two inequalities occur if and only if $\hat{R}$ satisfies $S_{d-1}$ and is equidimensional.

If $\left(^{*}\right)$ occurs then clearly $(\hat{R})_{p}$ must be Cohen-Macaulay for all $p \neq \hat{m}$, and since depth $\hat{R}=d-1$, this shows $\hat{R}$ satisfies $S_{d-1}$. Since we must have

$$
\operatorname{dim}(\hat{R})_{p}=\operatorname{dim} S_{p}-\operatorname{dim} S+\operatorname{dim} \hat{R}
$$

in this case, the work above shows that for all $p \supseteqq I$,

$$
\operatorname{dim} \hat{R}=\operatorname{dim} S / p+\operatorname{dim}(\hat{R})_{p},
$$

and this shows $\hat{R}$ is equidimensional.

Conversely, since $\hat{R}$ is catenary, if $\hat{R}$ satisfies $S_{d-1}$ and is equidimensional then

$$
\operatorname{depth}(\hat{R})_{p}=\operatorname{dim}(\hat{R})_{p}
$$

for all primes $p \neq \hat{m}$, and 


$$
\operatorname{dim} \hat{R}=\operatorname{dim} S / p+\operatorname{dim}(\hat{R})_{p}
$$

for all primes $p$. Thus in this case $\left(^{*}\right)$ holds and so $H_{m}^{d-1}(R)$ has finite length.

We now show (2) if and only if (3). Assume (2). Then there is a $N$ such that $m^{N} H_{m}^{d-1}(R)=0$. It was shown in [2] that if $R \rightarrow S$ faithfully flat and $x_{1}, \cdots, x_{n} \in R$ then these elements form a $d$-sequence in $R$ if and only if they form a $d$-sequence in $S$. Thus we may work in $\hat{R}$ and assume $R$ is complete for the remainder of this implication. By (1), $R$ is locally Cohen-Macaulay on the punctured spectrum, i.e., $R$ satisfies Serre's condition $S_{d-1}$.

Now let $x_{1}, \cdots, x_{d}$ be in $R$ such that ht $\left(x_{j_{1}}, \cdots, x_{j_{i}}\right)=i$ for each $i, 1 \leqq i \leqq d$.

Then since $R$ satisfies $S_{d-1}, x_{i_{1}}, \cdots, x_{i_{d-1}}$ form an $R$-sequence for any $d-1$ of $\left\{x_{1}, \cdots, x_{d}\right\}$. Hence to show (3) it is enough to show for $m_{i} \geqq N$ that

$$
\left(\left(x_{1}^{m_{1}}, \cdots, \hat{x}_{i}, \cdots, x_{d}^{m_{d}}\right): x_{i}^{2 m_{i}}\right)=\left(\left(x_{1}^{m_{1}}, \cdots, \widehat{x}_{i}, \cdots, x_{d}^{m} d\right): x_{i}^{m_{i}}\right) .
$$

Since we may rearrange the $x_{i}$ we may assume $i=d$. Suppose $\left(r_{1}, \cdots, r_{d}\right)$ is a syzygy of $\left(x_{1}^{m_{1}}, \cdots, x_{d}^{m}-_{1}^{-1}, x_{d}^{2 m_{d}}\right)$. Since $m^{N} H_{m}^{d-1}(R)=0$ we see that $x_{d}^{m} d$ must kill the image of this syzygy in $H_{m}^{d-1}(R)$.

By the construction (5) above we see this means that

$$
\left(r_{1} x_{d}^{m} d\left(x_{2}, \cdots, x_{d}\right)^{M}, \cdots, r_{d} x_{d}^{m} d\left(x_{1}, \cdots, x_{d-1}\right)^{M}\right)
$$

becomes a trivial syzygy of

$$
\left(x_{1}^{m_{1}+M}, \cdots, x_{d}^{m} \underline{d}_{-1}+3 L, x_{d}^{2 m} d_{d}^{+M}\right) .
$$

In particular,

$$
r_{d} x_{d}^{m}\left(x_{1}, \cdots, x_{d-1}\right)^{M} \in\left(x_{1}^{m_{1}+M}, \cdots, x_{d-1}^{m}{ }_{-1}{ }^{+M}\right) .
$$

As $x_{1}, \cdots, x_{d-1}$ forms an $R$-sequence, this shows (see [4]) that

$$
r_{d} x_{d}^{m} \in\left(x_{1}^{m_{1}}, \cdots, x_{d-1}^{m} \underline{d}^{-1}\right)
$$

which shows (3).

Now assume (3) and let us show (2). First, we show,

Lemma 1.1. Let $(R, m)$ be a local Noetherian ring of dimension. d. Suppose for every $x_{1}, \cdots, x_{d}$ in $m$ such that height $\left(x_{1}, \cdots, x_{j}\right)=j$, there exist integers $m_{1}, \cdots, m_{d} \geqq 1$ such that $x_{1}^{m_{1}}, \cdots, x_{d}^{m_{d}}$ form a $d$ sequence. Then $R_{p}$ is Cohen-Macaulay for all $p \neq m$.

Proof. Let $p$ be a minimal prime in $R$ with $R_{p}$ not CohenMacaulay. If height $p=n$, choose $a_{1}, \cdots, a_{n}$ in $p$ such that height 
$\left(a_{1}, \cdots, a_{i}\right)=i$. Complete $a_{1}, \cdots, a_{n}$ to a system of parameters $a_{1}, \cdots, a_{n}, a_{n+1}, \cdots, a_{d}$ of $R$ with ht $\left(a_{1}, \cdots, a_{i}\right)=i$. Since $p$ is the minimal prime which is not Cohen-Macaulay, we may assume $p$ is associated to $\left(a_{1}, \cdots, a_{i}\right)$ with $i<n$. Let $m_{1}, \cdots, m_{d}$ be chosen so that $a_{1}^{m_{1}}, \cdots, a_{d}^{m_{d}}$ form a $d$-sequence. Then $p$ is still associated to $a_{1}^{m_{1}}, \cdots, a_{i}^{m_{i}}$. By [3],

$$
\left(a_{1}^{m_{1}}, \cdots, a_{i}^{m_{i}}\right)=\left(\left(a_{1}^{m_{1}}, \cdots, a_{i}^{m_{i}}\right): a_{i+1}^{m_{i+1}}\right) \cap\left(a_{1}^{m_{1}}, \cdots, a_{d}^{m_{d}}\right) .
$$

Now since $\left(a_{1}^{m_{1}}, \cdots, a_{d}^{m_{d}} d\right)$ is primary to $m$, this decomposition shows that $p$ is associated to $\left(\left(a_{1}^{m_{1}}, \cdots, a_{i}^{m_{i}}\right): a_{i+1}^{m_{i+1}}\right)$. However $a_{i+1}^{m_{i+1}} \in p$ and $a_{i+1}^{m_{i+1}}$ is not a zero divisor modulo $\left(\left(a_{1}^{m_{1}}, \cdots, a_{i}^{m_{i}}\right): a_{i+1}^{m_{i+1}}\right)$. This contradiction proves the lemma.

Now assume (3). By Lemma $1.1 R$ satisfies $S_{d-1}$. (Note we may not assume $\hat{R}$ satisfies $S_{d-1}$ !)

Hence if $x_{1}, \cdots, x_{d}$ are chosen so that height $\left(x_{j_{1}}, \cdots, x_{j_{i}}\right)=i$ for all $1 \leqq i \leqq d$, to show $H_{m}^{d-1}(R)=0$ it is enough to show in this case that if such an $x_{1}, \cdots, x_{d}$ are a $d$-sequence, then

$$
\operatorname{syz}\left(x_{1}, \cdots, x_{d}\right) \longrightarrow \operatorname{syz}\left(x_{1}, \cdots, x_{d-1}, x_{d}^{2}\right)
$$

is onto. For if we can show this, then it is clear that the map

$$
\operatorname{syz}\left(x_{1}^{N}, \cdots, x_{d}^{N}\right) \longrightarrow H_{m}^{d-1}(R)
$$

will be onto, where $N$ is as in (3). This will show $H_{m}^{d-1}(R)$ is finitely generated; as $H_{m}^{d-1}(R)$ satisfies the descending chain condition, this will show (2).

So let $\left(r_{1}, \cdots, r_{d}\right)$ be a syzygy of $x_{1}, \cdots, x_{d-1}, x_{d}^{2}$. Then since

$$
r_{d} \in\left(\left(x_{1}, \cdots, x_{d-1}\right): x_{d}^{2}\right)=\left(\left(x_{1}, \cdots, x_{d-1}\right): x_{d}\right)
$$

we see

$$
\begin{gathered}
0=r_{d} x_{d}+\sum_{j=1}^{d-1} s_{i} x_{i}, \quad \text { and hence } \\
\left(r_{1}-s_{1} x_{d}\right) x_{1}+\cdots+\left(r_{d-1}-s_{d-1} x_{d}\right) x_{d-1}=0 .
\end{gathered}
$$

Thus, $\left(r_{1}-s_{1} x_{d}, \cdots, r_{d-1}-s_{d-1} x_{d}, 0\right)$ is a syzygy of $\left(x_{1}, \cdots, x_{d-1}, x_{d}^{2}\right)$. Since $x_{1}, \cdots, x_{d-1}$ will form an $R$-sequence, this syzygy of $\left(x_{1}, \cdots\right.$, $\left.x_{d-1}, x_{d}^{2}\right)$ will be trivial. Hence the image of $\left(s_{1}, \cdots, s_{d-1}, r_{d}\right)$ in $\operatorname{syz}\left(x_{1}, \cdots, x_{d}\right)$ will map onto $\left(r_{1}, \cdots, r_{d}\right) \in \operatorname{syz}\left(x_{1}, \cdots, x_{d}^{2}\right)$. This finishes the proof of Theorem 1.1.

Finally, we wish to relate condition (2) of Theorem 1.1 to the finiteness of $R^{(1)}$. To this end, let $(R, m)$ be a 2-dimensional Noetherian local domain and let $S=R^{(1)}=\bigcap R_{p}$ taken over all height one primes p. If $t$ is in $S$, then $J=\{r \in R \mid r t \in R\}$ is not contained in any height one prime and is thus primary to $m$. Hence if $x, y$ is an s.o.p., $x^{k} \in J$ for some $k$. Then $x^{k} t=r \in R$ and so $t=r / x^{k}$. Thus $J=\left(x^{k}: r\right)$ 
is primary to $m$, and so $y^{m} \in J$ for some $J$ which shows $r \in\left(x^{k}: y^{m}\right)$ for some $m$. Thus (see McAdam [7]), $S=\left\{r / x^{k} \mid r \in\left(x^{k}: y^{m}\right)\right.$ some $\left.k, m\right\}$. (The converse is easy to see; i.e., such $r / x^{k}$ are indeed in $R_{p}$ for all height one primes $p$.)

Now $H_{m}^{1}(R)$ in this case is the middle homology of

$$
R \longrightarrow R_{x} \oplus R_{y} \longrightarrow R_{x y} \longrightarrow 0
$$

That is, if

$$
\left\{\left(r / x^{k}, s / y^{e}\right) \mid r / x^{k}-s / y^{e}=0\right\}=N
$$

and $M=\{(r, r) \mid r \in R\}$ then

$$
H_{m}^{1}(R) \cong N / M
$$

(Note $r / x^{n}+s / y^{e}=0$ if and only if $r y^{e}+s x^{k}=0$ since $R$ is a domain.)

We map $S$ onto $H_{m}^{1}(R)$ as follows: if $t \in S$, let $g(t)=(t, t) \in N / M$. The discussion above shows $t \in R_{x} \cap R_{y}$ and so the map $g\left({ }_{-}\right)$makes sense. This map is clearly onto since

$$
S=\left\{r / x^{k} \mid r \in\left(x^{k}: y^{m}\right) \text { for some } k, m\right\}
$$

The kernel is the set of $t \in S$ such that $(t, t) \in M$; this is precisely if $t \in R$.

We have therefore shown

$$
H_{m}^{1}(R) \cong S / R
$$

Now if $S$ is finitely generated over $R$, then $H_{m}^{1}(R)$ is also and so it has finite length. Conversely, if $H_{m}^{1}(R)=S / R$ has finite length, then $S$ is obviously a finite $R$-module.

\section{REFERENCES}

1. A. Grothendieck, (Notes by R. Hartshorne) Local Cohomology Lecture notes in Math., no. 41, Springer-Verlag, Berlin, 1967.

2. — Element de geometrie algebrique, IV, I.H.E.S. Vol. 24.

3. C. Huneke, The theory of d-sequences and powers of ideals, (to appear, Advances in Mathematics).

4. I. Kaplansky, R-sequences and homological dimension, Nagoya Math. J., 20 (1962), 195-199.

5. E. Matlis, Injective modules over Noetherian rings, Pacific J. Math., 8 (1958), 511-528.

6. H. Matsumura, Commutative Algebra, W. A. Benjamin Inc., New York, 1970.

7. S. McAdam, Unmixed 2-dimensional local domains, Pacific J. Math., 68 (1977), 153-160.

8. L. J. Ratliff, A theorem on prime divisors of zero and characterizations of unmixed local domains, Pacific J. Math., 65 (1976), 449-470.

9. P. Samuel, Séminaire d'algébrae commutative, Anneaux de Gorenstein et torsion en algébre commutative, Secrétariat mathématique, 11, rue Pierre Curie, Paris (5), 1967. 
10. J. Stuckrod and W. Vogel, Towards a theory of Buchsbaum singularities, Amer. J. Math., 100 (1978), 727-746.

Received October 18, 1979. Partially supported by the National Science Foundation. UNIVERSITY OF MICHIGAN ANN ARBOR, MI 48109 


\section{PACIFIC JOURNAL OF MATHEMATICS}

\section{EDITORS}

DONALD BABBITT (Managing Editor)

University of Galifornia

Los Angeles, California 90024

Hugo RossI

University of Utah

Salt Lake City, UT 84112

C. C. MOORE AND ANDREW OGG

University of California

Berkeley, CA 94720

\section{J. DugundjI}

Department of Mathematics University of Southern California

Los Angeles, California 90007

R. Finn AND J. Milgram

Stanford University

Stanford, California 94305

\section{ASSOCIATE EDITORS}

R. ARENS

E. F. BECKENBACH

B. H. NeUmanN

F. WOLF

K. YosHIDA

\section{SUPPORTING INSTITUTIONS}

UNIVERSITY OF ARIZONA

UNIVERSITY OF BRITISH COLUMBIA

CALIFORNIA INSTITUTE OF TECHNOLOGY

UNIVERSITY OF CALIFORNIA

MONTANA STATE UNIVERSITY

UNIVERSITY OF NEVADA, RENO

NEW MEXICO STATE UNIVERSITY

OREGON STATE UNIVERSITY
UNIVERSITY OF OREGON

UNIVERSITY OF SOUTHERN CALIFONIA

STANFORD UNIVERSITY

UNIVERSITY OF HAWAII

UNIVERSITY OF TOKYO

UNIVERSITY OF UTAH

WASHINGTON STATE UNIVERSITY

UNIVERSITY OF WASHINGTON 


\section{Pacific Journal of Mathematics}

\section{Vol. 94, No. 1 \\ May, 1981}

Willy Brandal, Conditions for being an FGC domain $\ldots \ldots \ldots \ldots \ldots \ldots \ldots$

Allan Calder and Frank Williams, Incompressibility of maps and the

homotopy invariance of Čech cohomology $\ldots \ldots \ldots \ldots \ldots \ldots \ldots \ldots \ldots$

Jacques Chaumat, Quelques propriétés du couple d'espaces vectoriels

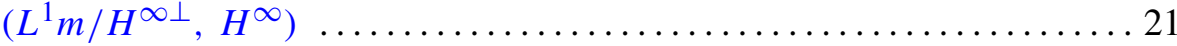

Manfred Droste and Rüdiger Göbel, Products of conjugate permutations . . 47

Jean Esterle, Rates of decrease of sequences of powers in commutative

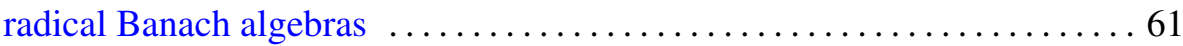

Allan Fryant, Ultraspherical expansions and pseudo analytic functions . . . 83

John Hannah, Homogenization of regular rings of bounded index. II . . . . . 107

Shigeru Haruki, On the theorem of S. Kakutani-M. Nagumo and J. L. Walsh

for the mean value property of harmonic and complex polynomials $\ldots .113$

Hugh M. Hilden, Representations of homology 3-spheres $\ldots \ldots \ldots \ldots \ldots 125$

Craig Huneke, A characterization of locally Macaulay completions . . . . . 131

Takesi Isiwata, Closed ultrafilters and realcompactness ................. 139

Joseph Weston Kitchen, Jr. and David A. Robbins, Tensor products of

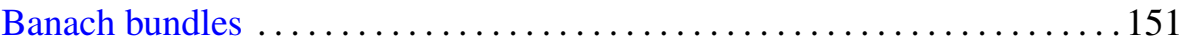

Allan J. Kroopnick, Note on bounded $L^{p}$-solutions of a generalized Liénard

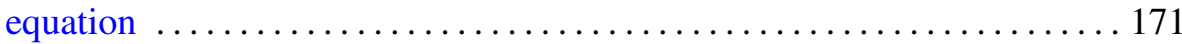

Ajay Kumar and Ajit Kaur Chilana, Spectral synthesis in products and quotients of hypergroups

Charles Livingston, Homology cobordisms of 3-manifolds, knot

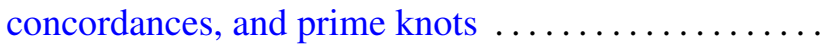

Hans Opolka, Projective representations of finite groups in cyclotomic fields

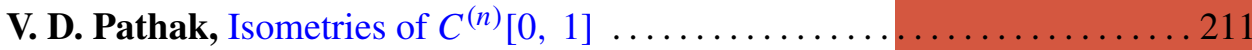

Mark Allan Pinsky, On the spectrum of Cartan-Hadamard manifolds . . . . . 2223

Judith Roitman, The number of automorphisms of an atomic Boolean

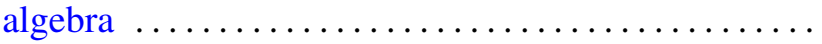

Kai Wang, Locally smooth torus group actions on integral cohomology complex projective spaces 